\title{
The rate of the molecular clock and the cost of gratuitous protein synthesis
}

\author{
Germán Plata ${ }^{1,2}$, Max E Gottesman ${ }^{3,4}$, Dennis Vitkup ${ }^{1,5^{*}}$
}

\begin{abstract}
Background: The nature of the protein molecular clock, the protein-specific rate of amino acid substitutions, is among the central questions of molecular evolution. Protein expression level is the dominant determinant of the clock rate in a number of organisms. It has been suggested that highly expressed proteins evolve slowly in all species mainly to maintain robustness to translation errors that generate toxic misfolded proteins. Here we investigate this hypothesis experimentally by comparing the growth rate of Escherichia coli expressing wild type and misfolding-prone variants of the LacZ protein.

Results: We show that the cost of toxic protein misfolding is small compared to other costs associated with protein synthesis. Complementary computational analyses demonstrate that there is also a relatively weaker, but statistically significant, selection for increasing solubility and polarity in highly expressed E. coli proteins.

Conclusions: Although we cannot rule out the possibility that selection against misfolding toxicity significantly affects the protein clock in species other than E. coli, our results suggest that it is unlikely to be the dominant and universal factor determining the clock rate in all organisms. We find that in this bacterium other costs associated with protein synthesis are likely to play an important role. Interestingly, our experiments also suggest significant costs associated with volume effects, such as jamming of the cellular environment with unnecessary proteins.
\end{abstract}

\section{Background}

Once the first protein sequences became available, their comparison led to the conclusion that the number of accumulated substitutions between orthologs was mainly a function of the evolutionary time elapsed since the last common ancestor of corresponding species [1,2]. Consequently, orthologous proteins accumulate substitutions at an approximately constant rate over long evolutionary intervals. This observation suggests that one can use available protein sequences as a molecular clock to estimate divergence times between different species [3]. Further studies revealed that while the pace of the molecular clock is similar for orthologous proteins in different lineages, it varies by several orders of magnitude across non-orthologous proteins $[4,5]$.

For several decades the dominant hypothesis explaining the large variability of the molecular clock rate between non-orthologous proteins was based on the

\footnotetext{
* Correspondence: dv2121@columbia.edu

${ }^{1}$ Center for Computational Biology and Bioinformatics, Columbia University,

1130 St Nicholas Ave, New York City, NY 10032, USA

Full list of author information is available at the end of the article
}

concept of functional protein density: the higher the fraction of protein residues directly involved in its function, the slower the protein molecular clock [6,7]. It was not until high-throughput genomics data became widely available that multiple molecular and genetic variables were used to investigate the dominant factors influencing the molecular clock rates of different proteins. Surprisingly, such features as gene essentiality [8-11], the number of protein-protein interactions [12,13], and specific functional roles $[14,15]$, have been shown to have, on average, either non-significant or significant but relatively weak correlations with protein evolutionary rates. On the other hand, quantities directly related to gene expression, such as codon bias, mRNA expression, and protein abundance, showed the strongest correlation with the rate of protein evolution [16,17]. For example, expression alone explains about a third of the variance in the substitution rates in several microbial species $[14,17,18]$ and about a quarter of the variance in Caenorhabditis elegans [19]. In these and many other organisms, highly expressed genes accept significantly less synonymous and non-synonymous (amino acid 
changing) substitutions than genes with low expression levels [20].

Considering the major role played by expression in setting the rate of amino acid substitutions, it is important to understand the main molecular mechanisms of this effect [21]. A popular theory by Drummond et al. $[18,22,23]$ suggests that highly expressed proteins may evolve slowly in all organisms, from microbes to human [22], due to the selection against toxicity associated with protein misfolding. The logic behind this interesting hypothesis is that a significant fraction $(>10 \%)$ of cellular proteins may contain translation errors $[24,25]$ that could cause cytotoxic protein misfolding. If misfolded proteins indeed incur substantial toxicity costs, greater pressure to avoid misfolding will affect highly expressed genes since they generate relatively more misfolded proteins [18]. Consequently, adaptive pressure will maintain sequences of highly expressed proteins robust to translation errors, which will in turn slow the amino acid substitution rate, that is, the protein molecular clock. The misfolding toxicity hypothesis was supported by the results of computer simulations [22], but to the best of our knowledge, it has never been tested experimentally.

In this study we specifically investigated whether the toxicity of misfolded proteins or other costs associated with protein synthesis make a dominant contribution to cellular fitness (growth rate), and consequently constrain the molecular clock in Escherichia coli. To test this, we used wild type (WT) and misfolding-prone variants of the E. coli $\beta$-galactosidase gene, lacZ. We also computationally analyzed the contribution of other related factors, such as protein stability and solubility.

\section{Results}

The native biological function of the LacZ protein is to cleave lactose for use as a source of carbon and energy [26]; in the absence of lactose, $\beta$-galactosidase does not participate in E. coli carbon metabolism. Therefore, we used lacZ expression in a lactose-free medium to measure the cost of gratuitous protein expression $[27,28]$. To compare that expression cost to the cost of potentially toxic protein misfolding, we used site-directed mutagenesis to engineer several destabilizing single-residue substitutions into LacZ. Single amino acid substitutions should serve as a good model for translational errors because only rarely, in about $10 \%$ of the proteins that contain translation errors, two or more residues will be simultaneously mistranslated in the same protein. We expressed the misfolding-prone mutants at the same level as the WT protein. Because the misfolded LacZ proteins are both potentially toxic and also devoid of biological function, the comparison of the growth rates of bacteria carrying the WT and each of the destabilized mutants allowed us to evaluate the additional fitness cost specifically arising from misfolding toxicity.

\section{Destabilizing mutations in lacZ yield aggregated and partialy soluble proteins}

Amino acid substitutions in protein cores are significantly more destabilizing than substitutions on protein surfaces $[29,30]$. Therefore, we selected five buried residues encoding non-polar amino acids that could be mutated to polar residues with single nucleotide substitutions while maintaining a similar level of codon preference (Table 1). We used the DPX server [31] to identify buried residues of the LacZ protein based on its crystal structure (Protein Data Bank (PDB) code 1dp0). We then applied the I-Mutant2.0 algorithm [32] to confirm that the selected substitutions would be indeed destabilizing. Using site-directed mutagenesis, the five selected substitutions were introduced separately into plasmids containing lac $Z$ under transcriptional control of the isopropyl $\beta$-D-1-thiogalactopyranoside (IPTG)inducible lac promoter [33]. We then used a $\beta$ galactosidase assay [34] to experimentally confirm reductions in the catalytic activity of LacZ in all of the generated mutants (Table 1).

To determine whether the destabilized proteins tended to aggregate, we separated soluble proteins and proteins in inclusion bodies (see Materials and methods) and analyzed them by SDS-PAGE (Figure 1a). The three mutants with the lowest catalytic activity (F758S, I141N and G353D) were found in inclusion bodies (Table 1), the remaining two mutants (V567D and A880E) and WT proteins were found mainly in the soluble protein fraction. Next, by inspecting total cell extracts at different time points after IPTG induction, we confirmed that the total amount of protein synthesized in each mutant strain was similar to that in the WT. As shown in Figure $1 \mathrm{~b}$, similar amounts of $\mathrm{LacZ}$ are produced in the WT and either soluble (V567D) or insoluble (F758S) mutants. Quantitative analysis of the Coomasie stained bands also did not reveal any significant difference between the LacZ synthesis rates in WT and mutant strains (Figure 1c). Finally, because expression of misfolded proteins is expected to generate a heat shock response $[35,36]$, we used western blots to monitor the amount of the GroEL heat shock protein in induced and un-induced cells carrying WT and mutant lacZ (Figure 1d). In cells carrying WT lacZ, the concentration of GroEL increased when IPTG was added. However, in both the V567D and F758S mutants, the levels of GroEL in either induced or uninduced cells were equal or higher than that in induced WT cells.

Overall, the results described in this section demonstrate that: all engineered mutants have significantly reduced catalytic activities; soluble and insoluble 
Table 1 Characteristics of destabilizing mutations engineered into $E$. coli $\beta$-galactosidase

\begin{tabular}{lccccc}
\hline & \multicolumn{4}{c}{ Mutant } \\
\cline { 2 - 6 } & V567D & F758S & I141N & G353D & A880E \\
\hline Predicted $\Delta \Delta \mathrm{G}(\mathrm{kcal} / \mathrm{mol})$ & -2.6 & -2.9 & -2.4 & -1.6 & -0.6 \\
Relative protein activity (\%) & 31 & 4 & 17 & 2 & 61 \\
Codon substitution $(\mathrm{WT} /$ mutant) & GTC/GAC & TTT/TCT & ATT/AAT & GGC/GAC & GCG/GAG \\
Codon preference \% (WT/mutant) & $13.5 / 53.9$ & $29.0 / 32.4$ & $33.5 / 17.3$ & $42.8 / 53.9$ & $32.3 / 24.7$ \\
Found in inclusion bodies (see Figure 1a) & No & Yes & Yes & Yes & No \\
\hline
\end{tabular}

In the table, $\Delta \Delta \mathrm{G}$ values represent destabilizing effects predicted by the I-Mutant2.0 server [32]. The experimentally determined enzymatic activities of the mutants (in percentages) are shown in the table relative to WT.

mutants are expressed at the same level as WT; and the mutants induce a heat shock response, and in some cases aggregate in inclusion bodies.

\section{Misfolded proteins are no more toxic than wild-type proteins}

The synthesis of WT or mutant $\beta$-galactosidase was initially induced by adding $10 \mu \mathrm{M}$ IPTG. Using WT LacZ activity as a reference [37], we estimated that about 30,000 molecules of $\beta$-galactosidase were present in each bacterial cell at this induction level. This approximately corresponds to half of the protein molecules expressed by a fully induced WT lacZ operon [34]. Cells expressing WT LacZ grew 13.5\% slower on glycerol as the sole carbon source compared to uninduced cells (Figure 2a). If misfolded proteins indeed impose a significant extra cost on the bacterium, then similarly expressed mutant strains with destabilizing

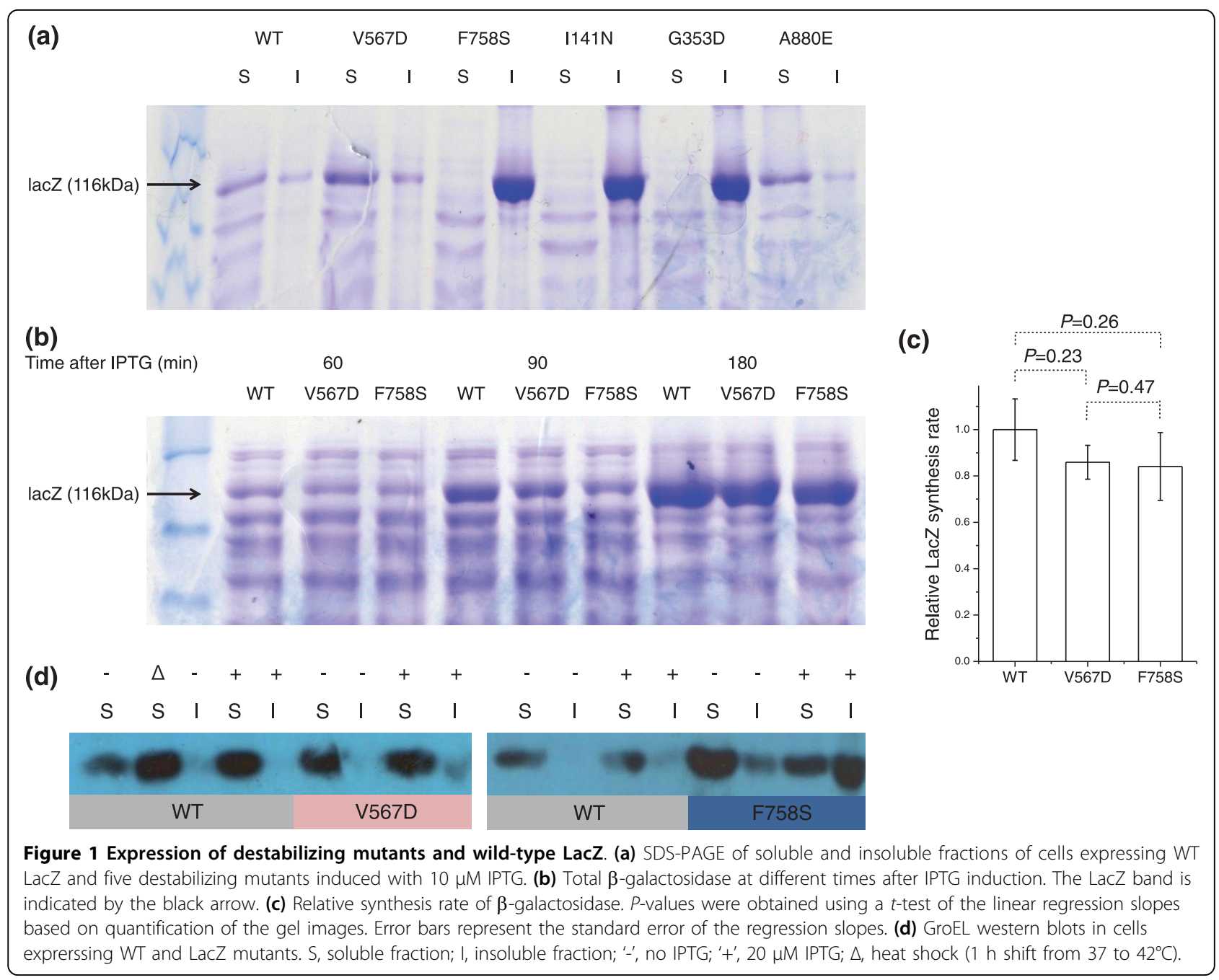




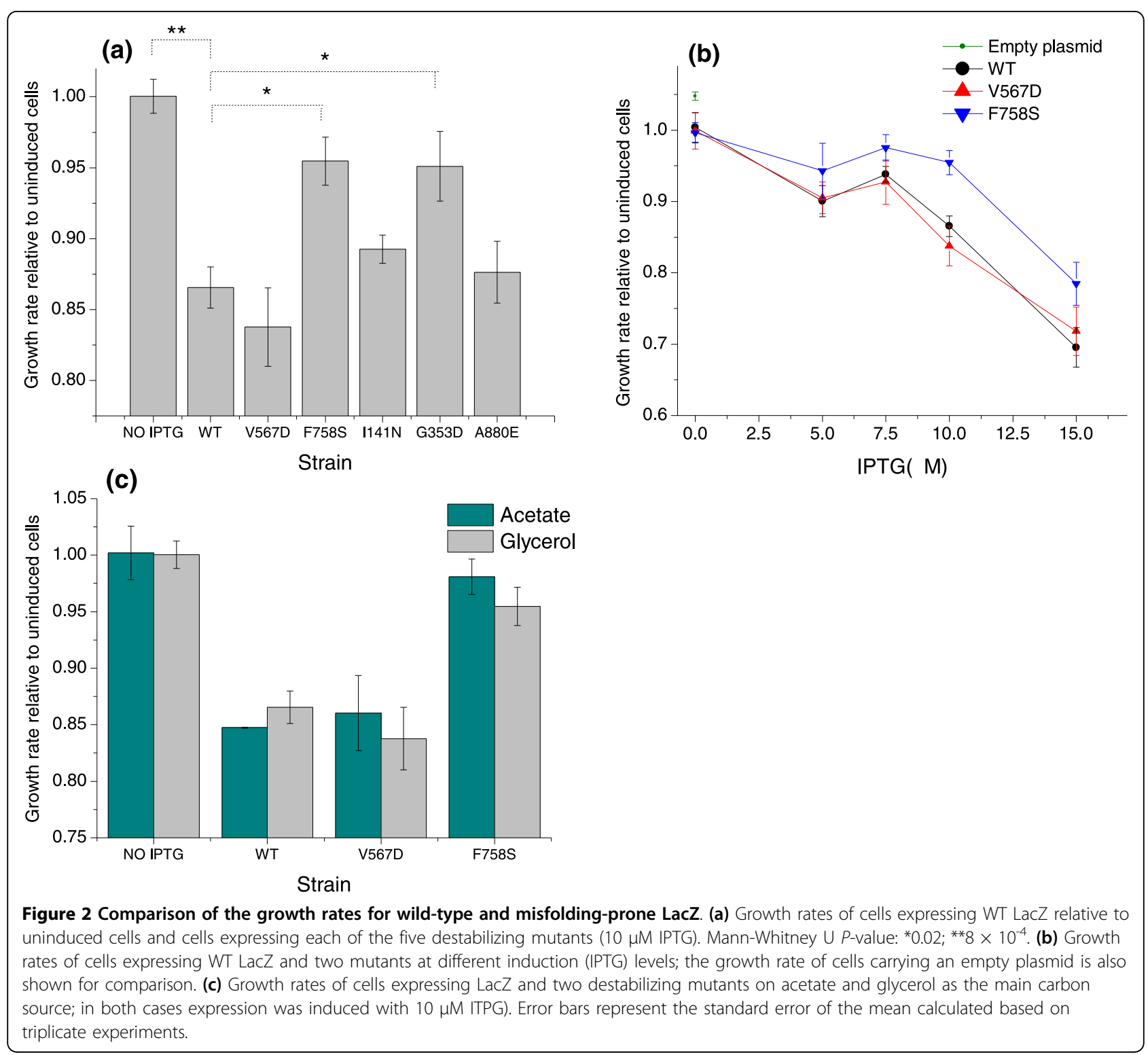

substitutions should lead to a more pronounced growth decrease compared to the one observed with WT LacZ. However, as shown in Figure $2 \mathrm{a}$, the mutant strains grew as well as cells expresing WT LacZ, and, despite inclusion body formation, two of the mutants even grew significantly faster (see Discussion).

To further explore the potential toxicity of the destabilized proteins, we focused on two mutants (F758S and V567D). These mutants are examples of a completely aggregated and a soluble but destabilized LacZ protein, respectively. By varying the concentration of IPTG, we monitored the growth of cells with different levels of expressed LacZ proteins (Figure 2b). Importantly, no additional growth decrease was observed in the mutant strains compared to the WT at all IPTG induction levels. When no IPTG was added, resulting in a low expression level from the un-induced promoter, we also observed the same growth rate reduction in all constructs relative to cells carrying an empty pBR322 plasmid (Figure 2b).

We investigated the possibility that the toxicity of misfolded proteins was more pronounced on a relatively poor carbon source by measuring the growth of the E. coli V567D and F758S mutants and the WT on acetate. Although the overall growth rate on acetate was only about $60 \%$ of that on glycerol, we again did not observe any additional fitness (growth) decrease due to the destabilizing mutations (Figure 2c). This experiment confirmed that the observed results are not specific to a particular carbon source. 
Nucleotide level selection, protein solubility, and stability in $E$. coli

Nucleotide sequences of highly expressed genes are significantly constrained by selection for amino acid codons corresponding to abundant tRNAs [38-40]. A recent experimental analysis by Kudla et al. [41] suggests that non-optimal codons can directly influence E. coli growth (fitness). Using 154 variants of GFP with multiple random synonymous substitutions, these authors found a significant positive correlation between codon optimality and bacterial growth rate. An important role played by the nucleotide-level selection in evolution of $E$. coli proteins is also supported by a high correlation between the rates of non-synonymous (Ka) and synonymous (Ks) substitutions (Figure 3b; Spearman's rank correlation $\mathrm{r}=0.66, P$-value $\left.<10^{-10}\right)$. In addition, the partial correlation between $\mathrm{Ka}$ and mRNA expression, controlling for $\mathrm{Ks}$, is small $(\mathrm{r}=-0.14, P=$ $7 \times 10^{-9}$ ), whereas the partial correlation between $\mathrm{Ks}$ and expression, controlling for $\mathrm{Ka}$, is significantly higher (r $\left.=-0.38, P<10^{-10}\right)$.

Although selection for optimal codons at the nucleotide level should significantly affect the rates of both synonymous and non-synonymous substitutions [40], there are additional constraints specifically acting on nonsynonymous sites $[42,43]$. Many of these additional constraints affect the propensity of proteins to misfold and aggregate. For example, it has been reported that highly expressed $E$. coli proteins are more soluble than proteins with lower expression [44-46]. It is likely that the observed increase in solubility is necessary to avoid protein aggregation and non-functional binding [47] mediated by non-

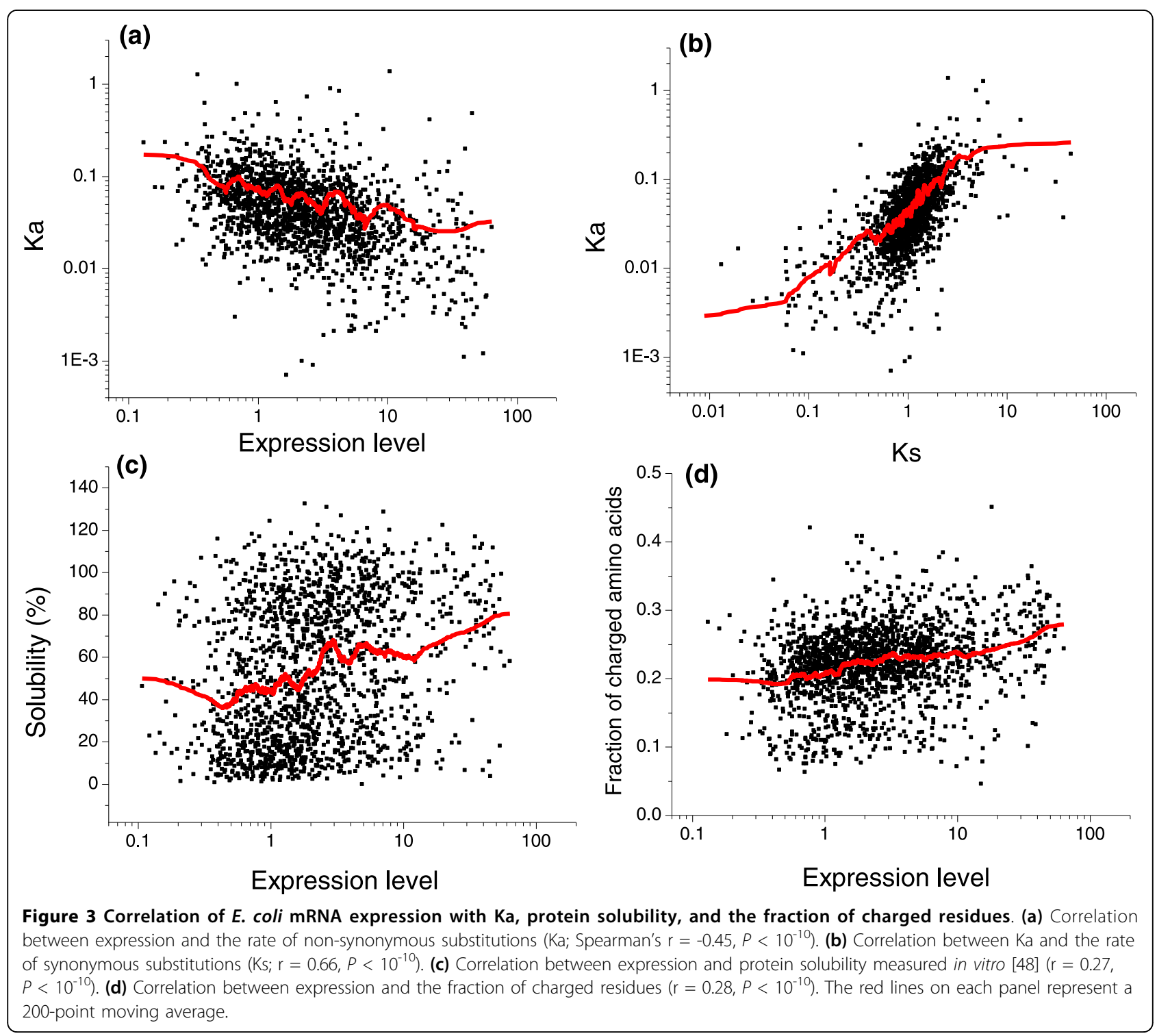


specific hydrophobic interactions. Using the genome-wide protein solubility data for $E$. coli proteins obtained by Niwa et al. [48], we indeed observed a significant correlation between solubility and expression (Figure 3c; Spearman's $r=0.27, P<10^{-10}$ ). Importantly, the observed selection for solubility does not explain the correlation between the protein evolutionary rate and expression (Figure 3a; $\mathrm{r}=-0.45, P<10^{-10}$ ); the partial correlation between $\mathrm{Ka}$ and expression, controlling either for solubility or for the fraction of charged residues, is still significant $(\mathrm{r}=$ -0.42 and -0.41 , respectively; $P<10^{-10}$ ).

The positive correlation between solubility and expression is in agreement with an increase in the fraction of charged residues (Figure 3d; $\mathrm{r}=0.28, P<10^{-10}$ ) and a simultaneous decrease in the fraction of hydrophobic residues $\left(\mathrm{r}=-0.16, P<10^{-10}\right)$ in highly expressed $E$. coli proteins. We observed similar results by analyzing $E$. coli protein duplicates (paralogs) with different expression levels. By directly comparing duplicates expressed at different levels, many confounding factors, such as differences in folding topology or protein secondary structure, are removed. The analysis of $370 \mathrm{E}$. coli paralogs (see Materials and methods) demonstrated a decrease in the fraction of hydrophobic residues (paired Wilcoxon signed rank test, $P=7 \times 10^{-4}$ ) and a simultaneous increase in the fraction of charged residues $\left(P=7 \times 10^{-6}\right)$ in the duplicates with higher expression levels.

The analysis of $602 \mathrm{E}$. coli protein structures currently available in the PDB (see Materials and methods) confirmed a significant increase in the fraction of solventexposed charged residues in highly expressed proteins $\left(\mathrm{r}=0.18, P=6 \times 10^{-6}\right)$. While such an increase may lead to higher protein stabilities [49], a proposed consequence of selection for translational robustness [22], we did not detect strong correlations between mRNA expression and other structural features usually associated with increased protein stability $[18,22]$. For example, we did not observe a significant increase in the fraction of buried hydrophobic residues $(r=0.06, P=0.13)$ [50-52] or an increase in the average number of contacts per residue (contact density) in highly expressed $E$. coli proteins $(\mathrm{r}=$ $0.02, P=0.96)$. Neither did we find a decrease in the fraction of residues in loops or unstructured protein regions ( $\mathrm{r}=0.07, P=0.06$ ) [53]. Our analysis of experimentally determined E. coli protein stabilities assembled in the ProTherm database [54] also failed to reveal any significant correlation between protein stability, measured either by protein melting temperature $(\mathrm{r}=-0.14, P=$ $0.46)$ or folding free energy $(\Delta \mathrm{G}, \mathrm{r}=-0.08, P=0.70)$, and mRNA expression level (Figure $4 \mathrm{a}, \mathrm{b})$. We also did not detect significant changes in the contact order, a structural measure strongly associated with folding speed $[55,56]$, in highly expressed bacterial proteins $(\mathrm{r}=-0.01$, $P=0.8)$.
Overall, the computational analysis described above suggests that, at least based on the currently available datasets, an increase in folding speed and/or protein stability for highly expressed bacterial proteins is unlikely to play a major role in constraining the protein molecular clock in E. coli.

\section{Discussion}

The results presented here demonstrate that, at least in $E$. coli, the cost associated with the gratuitous expression of a protein is significantly higher than the additional toxicity cost incurred by destabilization or misfolding of the same amount of protein; by 'gratuitous' we imply here that the protein has no effect on fitness through its biological function. It is important to emphasize that our growth measurements are not sensitive enough to detect small fitness effects - for example, decreases in the growth rate on the order of $1 \%$ or less - and consequently we cannot rule out additional costs specifically related to misfolding toxicity [57]. In fact, a detailed study by Lindner et al. [58] using time-lapse microscopy showed that the presence of protein aggregates in E. coli has an effect on growth rate at the level of individual cells. Nevertheless, our experiments do show that the misfolding toxicity cost is significantly smaller than other costs associated with protein expression.

We believe that the main expression costs specifically in this bacterium are related to translational efficiency and jamming of the cell's cytoplasm with useless proteins. Importantly, expression costs associated with amino acid waste, or the energy required for gratuitous expression, were recently shown by Stoebel et al. [59] to play a relatively minor role. On the other hand, both gratuitous protein expression and suboptimal codons can significantly slow bacterial growth, for instance, by reducing the pool of free ribosomes in the cell $[33,41]$. This effect will preferentially affect highly expressed genes bound by a relatively larger number of ribosomes. A gene with non-optimal codons will slow the rate of translation (speed of ribosomal motion) and thus titrate more ribosomes. A reduced pool of free ribosomes will necessarily slow expression of all bacterial genes and thus decrease the rate of biomass synthesis [60].

Interestingly, we observed that bacteria expressing two of the mutants (F758S and G353D) grew significantly faster than cells expressing native LacZ protein (Figure $2 a)$, although still not as fast as uninduced $E$. coli. This intriguing result demonstrates that titration of ribosomes cannot be the only explanation for the costs associated with gratuitous protein synthesis. The F758S and G353D proteins had the lowest catalytic activities of all constructs (Table 1) and both mutants, as well as I141N, were found mostly in inclusion bodies. It is likely that the localization of the LacZ proteins to inclusion 


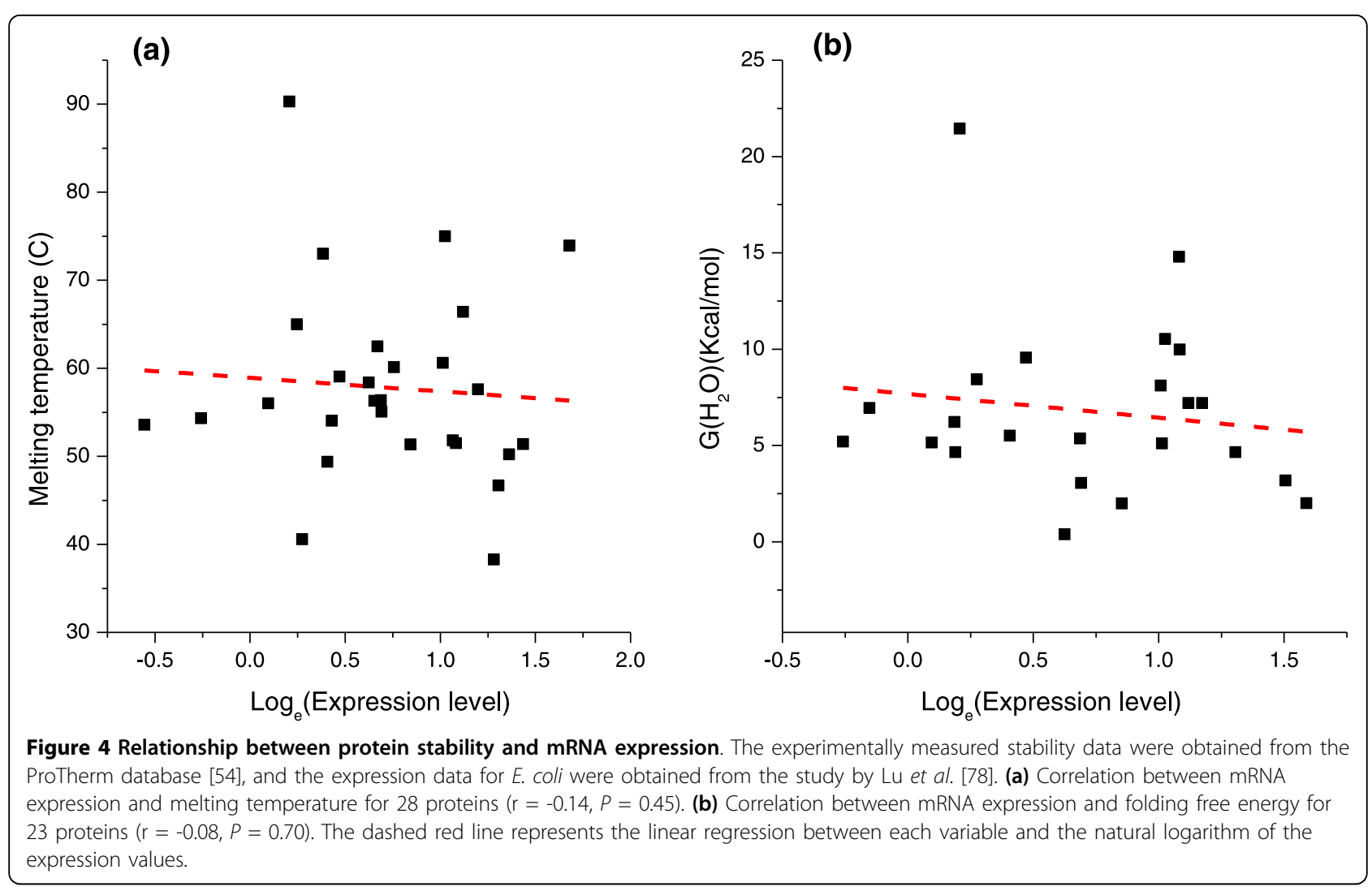

bodies prevents jamming of the cytoplasm and relieves effects associated with non-functional binding. It was previously shown that an asymmetric partition of inclusion bodies during cell division may result in a cell rejuvenation phenotype [58]. We would like to emphasize that this result does not support the misfolding toxicity hypothesis, as these mutants grew faster than the strain expressing WT LacZ. Based on the growth rates of mutants primarily localized to inclusion bodies (V567D, F758S, I141N; average growth decrease 6.7\%) and the proteins remaining in the cytoplasm (WT, V567D, A880E; average growth decrease 14\%), one can conclude that effects of jamming and translational efficiency make approximately similar contributions to fitness.

An important separate question in the context of the mistranslation-induced misfolding hypothesis is whether phenotypic (transcriptional or translational) mutations can cause enough protein misfolding to be significantly cytotoxic. Although suboptimal codons are expected to substantially increase the translational error rate [39], no correlation was observed between codon optimization and the fraction of properly folded GFP by Kudla et al. [41]. Even if relatively rare, phenotypic mutations can still be significantly damaging if they occur in functionally and structurally important sties. This may explain a well-established correlation between codon optimization and evolutionary conservation of corresponding protein sites [61-63]. This correlation is not necessarily a consequence of selection against mistranslation-induced toxicity, and again may be primarily related to the loss of functional proteins and the cost of additional protein synthesis necessary to compensate for the misfolding. In fact, it has been reported that essential bacterial proteins have lower aggregation propensities than those predicted for non-essential proteins [46].

While our study demonstrates that misfolding toxicity is unlikely to be a universally dominant factor connecting expression and the protein molecular clock in all species, we cannot rule out the possibility that toxicity may play an important role in other species. We note, however, that in higher organisms the correlations between mRNA expression and the protein molecular clock are generally much weaker than in some microbes. For example, Liao et al. [64] demonstrated that expression plays a relatively minor role in constraining the molecular clock in mammalian species. Also, by comparing evolutionary rate of separate and fused protein domains in human and Arabidopsis, Wolf et al. [65] found a comparable contribution from expression and structural-functional constraints.

A number of elegant experimental studies have demonstrated a cytotoxic effect of several misfolded or marginally 
stable proteins in higher organisms [66,67]. For instance, several hundred mutations in the SOD1 protein were shown to result in aggregates associated with amyotrophic lateral sclerosis in humans [68]; also, non-natural peptides have been used to induce cytotoxic aggregates of GFP in C. elegans [69]. Although these studies directly demonstrate the importance of misfolding and aggregation for some specific proteins, the extent to which these effects dominate the molecular clock for all proteins in these and other species needs to be investigated and again compared to other contributing factors.

\section{Conclusions}

Our experimental results suggest that selection against toxic protein misfolding is unlikely to be the universal and dominant factor determining the rate of the protein molecular clock in all species. We demonstrate that, at least in E. coli, other factors associated with gratuitous protein synthesis, such as translational efficiency and possibly jamming of the cytoplasm, are likely to be the primary constraints. Our computational analyses also suggest a relatively weaker, but statistically significant, selection for increasing solubility and polarity in highly expressed E. coli proteins.

\section{Materials and methods}

\section{Strains and mutant generation}

E. coli K12 strain GP4 (W3102, XA 21Z, lac $^{\mathrm{q}}$ ) was used in all experiments. lac $Z$ was expressed from the IPTGinducible lac promoter in plasmid PIV18 [33]; PIV18 is a pBR322 derivative that carries a mutation in the Shine Dalgarno sequence of the lacZ transcript that increases translation efficiency. Site directed mutagenesis was carried out using Stratagene's QuikChange Lightning kit (Stratagene, Cedar Creek, TX, USA). pBR322 was used as the empty plasmid control.

\section{Growth curve analysis}

For each construct, a sweep of colonies was grown overnight on Luria-Bertani (LB) liquid media supplemented with $100 \mu \mathrm{g} / \mathrm{ml}$ ampicillin. Overnight cultures were diluted by a 1:100 factor and grown on M9 minimal media suplemented with $0.5 \%$ casaminoacids, $0.25 \mu \mathrm{g} / \mathrm{ml}$ thiamine, $100 \mu \mathrm{g} / \mathrm{ml}$ ampicillin and either $0.4 \%$ glycerol or acetate as carbon sources. We transfered $300 \mu \mathrm{l}$ of cells with an OD600 of 0.5 to flasks containing $5.5 \mathrm{ml}$ of prewarmed media suplemented with the appropiate amount of IPTG. Two hours after induction, OD600 was measured every 45 minutes. Growth rate was determined as the regression line slope of time and the logarithm of OD600.

\section{SDS-PAGE and western blotting}

The equivalent of $200 \mu \mathrm{l}$ of cells at an OD600 of 0.7 was collected by centrifugation and lysed using Novagen's
BugBuster (primary amine-free) Protein Extraction Reagent (Novagen, Merck, Darmstadt, Germany). Soluble proteins were retrieved after centrifugation of the lysed cells and aggregated proteins were then harvested following instructions for inclusion body purification described in the BugBuster reagent manual. Both fractions were saved in a $50 \mu \mathrm{l}$ volume including $10 \mu \mathrm{l} 4 \times$ SDS loading buffer, boiled, and electrophoresed on a $10 \%$ SDS polyacrylamide gel. Gels were stained with Coomassie blue and scanned for analysis. For the analysis of total protein, cells were lysed in BugBuster reagent containing rLysozyme and boiled after addition of $4 \times$ SDS loading buffer. Bands were quantified using the ImageJ program [70].

Protein samples separated by SDS-PAGE as described above were blotted overnight onto a nitrocellulose membrane and incubated with Anti-GroEL antibody produced in rabbit 1:10 000 (Sigma Aldrich, St Louis, MO, USA). Blots were blocked with $5 \%$ non-fat dry milk, incubated with 1:3,000 anti-rabbit horseradish peroxidase conjugate antibody and visualized with Amersham's ECL Plus Western Blotting Reagent (GE Healthcare, Munich, Germany).

\section{Structural analysis of $E$. coli proteins}

In the analysis we used $602 \mathrm{E}$. coli protein structures currently available in the PDB [71]. To prevent sampling biases, we filtered available PDB entries so that no two protein structures used in the calculations had sequence identity higher than 90\%; similar results were obtained without filtering. We defined buried residues as those with a solvent accessible area smaller than 16\% [72,73]. Solvent accessibility was calculated by the DSSP [74] program. The fraction of protein residues in loops was also calculated using DSSP. Two non-adjacent protein residues were considered to be in contact if any two of their non-hydrogen atoms were closer than $4.5 \AA$ [75]. The protein contact density was defined as the average number of non-adjacent contacts per residue. Contact order was calculated as $(\mathrm{L} \times \mathrm{N})^{-1} \times \Sigma \Delta \mathrm{S}_{\mathrm{ij}}$, where $\mathrm{N}$ is the total number of contacts, $\mathrm{L}$ is the total number of residues in the protein and $\Delta S_{\mathrm{ij}}$, which is summed over all contacts, is the number of amino acids separating contacting residues [56]. In vitro solubility data for $E$. coli proteins was obtained directly from the study of Niwa et al. [48].

\section{Correlation of the synonymous (Ks) and non-synonymous} (Ka) substitution rates with expression

Orthologous open reading frames and protein sequences from E. coli and Salmonella enterica were used to calculate Ks and Ka values. The E. coli-Salmonella orthologs were determined as bi-directional best hits using protein BLAST [76]. Ka and Ks values were calculated using the 
maximum likelihood method implemented in the PAML package [77]. The mRNA expression data reported by Lu et al. [78] were used to calculate the correlations. For the analysis of duplicated genes, we defined duplicates as pairs of $E$. coli proteins having more than $40 \%$ sequence identity that could be aligned for at least $80 \%$ of their total length using BLAST. In the analysis of duplicates, we used expression data from 466 experiments in the Many Microbes Microarrays Database [79]. We selected for the analysis only the pairs for which one paralog had higher expression values in more than $80 \%$ of the reported experiments.

\section{Abbreviations}

GF: green fluorescent protein; IPTG: isopropyl $\beta$-D-1-thiogalactopyranoside; Ka: the rate of non-synonymous substitutions; Ks: the rate of synonymous substitutions; PDB: Protein Data Bank; WT: wild type.

\section{Acknowledgements}

We would like to thank Steen Pedersen (University of Copenhagen) for kindly providing the WT LacZ and pBR322 plasmids. We also thank Barry Honig for many helpful discussions on protein folding and stability. This work was supported in part by NIGMS grant GM079759 to DV, and the National Centers for Biomedical Computing U54CA121852 grant to Columbia University.

\section{Author details}

'Center for Computational Biology and Bioinformatics, Columbia University, 1130 St Nicholas Ave, New York City, NY 10032, USA. ${ }^{2}$ Integrated Program in Cellular, Molecular, Structural, and Genetic Studies, Columbia University, 1130 St Nicholas Ave, New York City, NY 10032, USA. ${ }^{3}$ Department of Microbiology and Immunology, Columbia University, 701 W. 168 St, New York City, NY 10032, USA. ${ }^{4}$ Department of Biochemistry and Molecular Biophysics, Columbia University, 701 W. 168 St, New York City, NY 10032, USA. ${ }^{5}$ Department of Biomedical Informatics, Columbia University, 1130 St Nicholas Ave, New York City, NY 10032, USA.

\section{Authors' contributions}

GP carried out the experiments and computational analyses. DV, MG and GP conceived the experiments and analyzed the results. DV and GP wrote the paper with revisions by MG. All authors read and approved the final manuscript.

\section{Competing interests}

The authors declare that they have no competing interests.

Received: 8 June 2010 Revised: 3 September 2010

Accepted: 29 September 2010 Published: 29 September 2010

\section{References}

1. Zuckerkandl E, Pauling L: Evolutionary divergence and convergence in proteins. In Evolving Genes and Proteins. Edited by: Bryson V, Vogel H. New York: Academic Press; 1965:97-166.

2. Margoliash E: Primary structure and evolution of cytochrome C. Proc Natl Acad Sci USA 1963, 50:672-679.

3. Benton MJ, Donoghue PC: Paleontological evidence to date the tree of life. Mol Biol Evol 2007, 24:26-53.

4. Gillespie JH: The Causes of Molecular Evolution New York: Oxford University Press 1991.

5. Ayala FJ: Molecular clock mirages. Bioessays 1999, 21:71-75

6. Zuckerkandl E: Evolutionary processes and evolutionary noise at the molecular level. I. Functional density in proteins. J Mol Evol 1976, 7:167-183

7. Wilson AC, Carlson SS, White TJ: Biochemical evolution. Annu Rev Biochem 1977, 46:573-639.
8. Wang Z, Zhang JZ: Why Is the correlation between gene importance and gene evolutionary rate so weak? PLoS Genet 2009, 5:e1000329.

9. Vitkup $D$, Kharchenko $P$, Wagner A: Influence of metabolic network structure and function on enzyme evolution. Genome Biol 2006, 7:R39.

10. Jordan IK, Rogozin IB, Wolf YI, Koonin EV: Essential genes are more evolutionarily conserved than are nonessential genes in bacteria. Genome Res 2002, 12:962-968.

11. Hirsh $A E$, Fraser $H B$ : Protein dispensability and rate of evolution. Nature 2001, 411:1046-1049.

12. Jordan IK, Wolf $\mathrm{Yl}$, Koonin EV: No simple dependence between protein evolution rate and the number of protein-protein interactions: only the most prolific interactors tend to evolve slowly. BMC Evol Biol 2003, 3:1.

13. Fraser HB, Hirsh AE, Steinmetz LM, Scharfe C, Feldman MW: Evolutionary rate in the protein interaction network. Science 2002, 296:750-752.

14. Rocha EP, Danchin A: An analysis of determinants of amino acids substitution rates in bacterial proteins. Mol Biol Evol 2004, 21:108-116.

15. Koonin EV: Systemic determinants of gene evolution and function. Mol Syst Biol 2005, 1:2005 0021.

16. Xia Y, Franzosa EA, Gerstein MB: Integrated assessment of genomic correlates of protein evolutionary rate. PLoS Comput Biol 2009, 5: e1000413.

17. Pal C, Papp B, Hurst LD: Highly expressed genes in yeast evolve slowly. Genetics 2001, 158:927-931.

18. Drummond DA, Bloom JD, Adami C, Wilke CO, Arnold FH: Why highly expressed proteins evolve slowly. Proc Natl Acad Sci USA 2005, 102:14338-14343.

19. Marais G, Duret L: Synonymous codon usage, accuracy of translation, and gene length in Caenorhabditis elegans. J Mol Evol 2001, 52:275-280.

20. Rocha EP: The quest for the universals of protein evolution. Trends Genet 2006, 22:412-416.

21. Pal C, Papp B, Lercher MJ: An integrated view of protein evolution. Nat Rev Genet 2006, 7:337-348.

22. Drummond DA, Wilke CO: Mistranslation-induced protein misfolding as a dominant constraint on coding-sequence evolution. Cell 2008, 134:341-352.

23. Drummond DA, Wilke CO: The evolutionary consequences of erroneous protein synthesis. Nat Rev Genet 2009, 10:715-724.

24. Kramer $E B$, Farabaugh $P J$ : The frequency of translational misreading errors in E. coli is largely determined by tRNA competition. RNA 2007, 13:87-96.

25. Stansfield I, Jones KM, Herbert P, Lewendon A, Shaw WV, Tuite MF: Missense translation errors in Saccharomyces cerevisiae. J Mol Biol 1998, 282:13-24

26. Müller-Hill B: The lac Operon: A Short History of a Genetic Paradigm. New York: Walter de Gruyter 1996.

27. Dong HJ, Nilsson L, Kurland CG: Gratuitous overexpression of genes in Escherichia coli leads to growth inhibition and ribosome destruction. $J$ Bacteriol 1995, 177:1497-1504.

28. Dekel $\mathrm{E}$, Alon U: Optimality and evolutionary tuning of the expression level of a protein. Nature 2005, 436:588-592.

29. Pakula AA, Sauer RT: Genetic analysis of protein stability and function Annu Rev Genet 1989, 23:289-310.

30. Matthews BW: Structural and genetic analysis of the folding and function of T4 lysozyme. FASEB J 1996, 10:35-41.

31. Vlahovicek K, Pintar A, Parthasarathi L, Carugo O, Pongor S: CX, DPX and PRIDE: WWW servers for the analysis and comparison of protein 3D structures. Nucleic Acids Res 2005, 33:W252-W254.

32. Capriotti E, Fariselli P, Casadio R: I-Mutant2.0: predicting stability changes upon mutation from the protein sequence or structure. Nucleic Acids Res 2005, 33:W306-W310.

33. Vind J, Sorensen MA, Rasmussen MD, Pedersen S: Synthesis of proteins in Escherichia coli is limited by the concentration of free ribosomes. Expression from reporter genes does not always reflect functional mRNA levels. J Mol Biol 1993, 231:678-688.

34. Sambrook J, Russell DW: Molecular Cloning: A Laboratory Manual Woodbury, NY; CSHL Press 2001.

35. Lesley SA, Graziano J, Cho CY, Knuth MW, Klock HE: Gene expression response to misfolded protein as a screen for soluble recombinant protein. Protein Eng 2002, 15:153-160.

36. Parsell DA, Sauer RT: Induction of a heat shock-like response by unfolded protein in Escherichia coli: Dependence on protein level not protein degradation. Genes Dev 1989, 3:1226-1232. 
37. Wang IN, Deaton J, Young R: Sizing the holin lesion with an endolysinbeta-galactosidase fusion. J Bacteriol 2003, 185:779-787.

38. Andersson SG, Kurland CG: Codon preferences in free-living microorganisms. Microbiol Rev 1990, 54:198-210.

39. Bulmer M: The selection-mutation-drift theory of synonymous codon usage. Genetics 1991, 129:897-907.

40. Akashi H: Gene expression and molecular evolution. Curr Opin Genet Dev 2001, 11:660-666.

41. Kudla G, Murray AW, Tollervey D, Plotkin JB: Coding-sequence determinants of gene expression in Escherichia coli. Science 2009, 324:255-258.

42. Pal C, Papp B, Lercher MJ: An integrated view of protein evolution. Nat Rev Genet 2006, 7:337-348.

43. Koonin EV, Wolf Yl: Constraints and plasticity in genome and molecularphenome evolution. Nat Rev Genet 2010, 11:487-498.

44. Dobson CM: Protein misfolding, evolution and disease. Trends Biochem SC 1999, 24:329-332.

45. Tartaglia GG, Pechmann S, Dobson CM, Vendruscolo M: A relationship between mRNA expression levels and protein solubility in E. coli. $J \mathrm{Mol}$ Biol 2009, 388:381-389.

46. de Groot NS, Ventura S: Protein aggregation profile of the bacterial cytosol. PLoS One 2010, 5:e9383.

47. Zhang J, Maslov S, Shakhnovich El: Constraints imposed by non-functiona protein-protein interactions on gene expression and proteome size. Mol Syst Biol 2008, 4:210.

48. Niwa T, Ying BW, Saito K, Jin W, Takada S, Ueda T, Taguchi H: Bimodal protein solubility distribution revealed by an aggregation analysis of the entire ensemble of Escherichia coli proteins. Proc Natl Acad Sci USA 2009, 106:4201-4206.

49. Strub C, Alies C, Lougarre A, Ladurantie C, Czaplicki J, Fournier D: Mutation of exposed hydrophobic amino acids to arginine to increase protein stability. BMC Biochem 2004, 5:9.

50. Baldwin RL: Energetics of protein folding. J Mol Biol 2007, 371:283-301.

51. Dill KA: Dominant forces in protein folding. Biochemistry 1990, 29:7133-7155.

52. Honig B, Yang AS: Free energy balance in protein folding. Adv Protein Chem 1995, 46:27-58

53. Thompson MJ, Eisenberg D: Transproteomic evidence of a loop-deletion mechanism for enhancing protein thermostability. J Mol Biol 1999, 290:595-604.

54. Bava KA, Gromiha MM, Uedaira H, Kitajima K, Sarai A: ProTherm, version 4.0: thermodynamic database for proteins and mutants. Nucleic Acids Res 2004, 32:D120-121.

55. Ivankov DN, Garbuzynskiy SO, Alm E, Plaxco KW, Baker D, Finkelstein AV: Contact order revisited: influence of protein size on the folding rate. Protein Sci 2003, 12:2057-2062.

56. Plaxco KW, Simons KT, Baker D: Contact order, transition state placement and the refolding rates of single domain proteins. J Mol Biol 1998, 277:985-994.

57. Thatcher JW, Shaw JM, Dickinson WJ: Marginal fitness contributions of nonessential genes in yeast. Proc Natl Acad Sci USA 1998, 95:253-257.

58. Lindner AB, Madden R, Demarez A, Stewart EJ, Taddei F: Asymmetric segregation of protein aggregates is associated with cellular aging and rejuvenation. Proc Natl Acad Sci USA 2008, 105:3076-3081.

59. Stoebel DM, Dean AM, Dykhuizen DE: The cost of expression of Escherichia coli lac operon proteins is in the process, not in the products. Genetics 2008, 178:1653-1660.

60. Tuller T, Waldman YY, Kupiec M, Ruppin E: Translation efficiency is determined by both codon bias and folding energy. Proc Natl Acad Sci USA 2010, 107:3645-3650.

61. Akashi H: Synonymous codon usage in Drosophila melanogaster: natural selection and translational accuracy. Genetics 1994, 136:927-935.

62. Huang Y, Koonin EV, Lipman DJ, Przytycka TM: Selection for minimization of translational frameshifting errors as a factor in the evolution of codon usage. Nucleic Acids Res 2009, 37:6799-6810.

63. Stoletzki N, Eyre-Walker A: Synonymous codon usage in Escherichia coli: selection for translational accuracy. Mol Biol Evol 2007, 24:374-381.

64. Liao BY, Scott NM, Zhang J: Impacts of gene essentiality, expression pattern, and gene compactness on the evolutionary rate of mammalian proteins. Mol Biol Evol 2006, 23:2072-2080.
65. Wolf MY, Wolf YI, Koonin EV: Comparable contributions of structuralfunctional constraints and expression level to the rate of protein sequence evolution. Biol Direct 2008, 3:40.

66. Bucciantini M, Giannoni E, Chiti F, Baroni F, Formigli L, Zurdo J, Taddei N, Ramponi G, Dobson CM, Stefani M: Inherent toxicity of aggregates implies a common mechanism for protein misfolding diseases. Nature 2002, 416:507-511.

67. Gidalevitz T, Ben-Zvi A, Ho KH, Brignull HR, Morimoto Rl: Progressive disruption of cellular protein folding in models of polyglutamine diseases. Science 2006, 311:1471-1474

68. Munch C, Bertolotti A: Exposure of hydrophobic surfaces initiates aggregation of diverse ALS-causing superoxide dismutase-1 mutants. $J \mathrm{Mol}$ Biol 2010, 399:512-525.

69. Link CD, Fonte V, Hiester B, Yerg J, Ferguson J, Csontos S, Silverman MA, Stein GH: Conversion of green fluorescent protein into a toxic, aggregation-prone protein by C-terminal addition of a short peptide. J Biol Chem 2006, 281:1808-1816.

70. Abramoff MD, Magelhaes PJ, Ram SJ: Image Processing with ImageJ. Biophotonics Int 2004, 11:36-42.

71. Berman HM, Westbrook J, Feng Z, Gilliland G, Bhat TN, Weissig H, Shindyalov IN, Bourne PE: The Protein Data Bank. Nucleic Acids Res 2000, 28:235-242.

72. Momen-Roknabadi A, Sadeghi M, Pezeshk H, Marashi SA: Impact of residue accessible surface area on the prediction of protein secondary structures. BMC Bioinformatics 2008, 9:357.

73. Rost B, Sander C: Conservation and prediction of solvent accessibility in protein families. Proteins 1994, 20:216-226.

74. Kabsch W, Sander C: Dictionary of protein secondary structure: pattern recognition of hydrogen-bonded and geometrical features. Biopolymers 1983, 22:2577-2637.

75. Bloom JD, Drummond DA, Arnold FH, Wilke CO: Structural determinants of the rate of protein evolution in yeast. Mol Biol Evol 2006, 23:1751-1761.

76. Altschul SF, Madden TL, Schaffer AA, Zhang J, Zhang Z, Miller W, Lipman DJ: Gapped BLAST and PSI-BLAST: a new generation of protein database search programs. Nucleic Acids Res 1997, 25:3389-3402.

77. Yang ZH: PAML: a program package for phylogenetic analysis by maximum likelihood. Comput Appl Biosci 1997, 13:555-556.

78. Lu P, Vogel C, Wang R, Yao X, Marcotte EM: Absolute protein expression profiling estimates the relative contributions of transcriptional and translational regulation. Nat Biotechnol 2007, 25:117-124.

79. Faith JJ, Driscoll ME, Fusaro VA, Cosgrove EJ, Hayete B, Juhn FS, Schneider SJ, Gardner TS: Many Microbe Microarrays Database: uniformly normalized Affymetrix compendia with structured experimental metadata. Nucleic Acids Res 2008, 36:D866-870.

doi:10.1186/gb-2010-11-9-r98

Cite this article as: Plata et al:: The rate of the molecular clock and the cost of gratuitous protein synthesis. Genome Biology 2010 11:R98.

\section{Submit your next manuscript to BioMed Central and take full advantage of:}

- Convenient online submission

- Thorough peer review

- No space constraints or color figure charges

- Immediate publication on acceptance

- Inclusion in PubMed, CAS, Scopus and Google Scholar

- Research which is freely available for redistribution 